\title{
A comment on the paper 'Biomarkers in Multiple Sclerosis' by Sapko et al.
}

\author{
Piotr Lewczuk ${ }^{1,2}$, Johannes Kornhuber ${ }^{1}$, Barbara Mroczko \\ ${ }^{1}$ Department of Psychiatry and Psychotherapy, Universitätsklinikum Erlangen, and Friedrich-Alexander Universität \\ Erlangen-Nürnberg, Erlangen, Germany \\ ${ }^{2}$ Department of Neurodegeneration Diagnostics, Medical University of Białystok, Białystok, Poland
}

(Neurol Neurochir Pol 2021; 55 (1): 115-116)

\section{To the Editors:}

We read with great interest the review paper recently published by Sapko et al. [1]. The article exhaustively presented current perspectives in the development of candidate multiple sclerosis (MS) biomarkers in cerebrospinal fluid (CSF), with very interesting concepts on their diagnostic/relevant interpretations.

Unfortunately, it was lacking in what it partly set out to do, namely a more extended review of the established, routine biomarkers of the disease, as well as a biophysical background as to how any new candidate biomarker should be evaluated. We believe this calls for comment.

MS is a chronic neuroinflammatory disease of autoimmune type. Therefore it is not surprising that it is diagnosed, at least neurochemically, with CSF biomarkers reflecting autoimmune neuroinflammation, particularly its chronic and polyspecific aspects.

(i) The most common neurochemical characteristic of chronic neuroinflammation is the intrathecal, polyspecific synthesis of immunoglobulins, particularly IgG, which is routinely assessed by a comparison of the immunoglobulins' CSF-serum concentration quotients $\left(\mathrm{Q}_{\mathrm{Ig}}\right)$ with the biomarker of the blood-CSF barrier function (not to be confused with the blood-brain barrier), the CSF-serum concentration quotient of albumin $\left(\mathrm{Q}_{\mathrm{Alb}}\right)$. This non-linear, hyperbolic association is best observed in a graphical form, on diagrams devised in the 1990s by H. Reiber [2], and since then known, after their inventor, as Reibergramms. Three of these exist, for the three routinely analysed CSF immunoglobulin classes: IgG, IgA, and IgM; MS characterises with either exclusive IgG synthesis, or its dominance, with c.20-30\% of MS patients having concomitant IgM and/or IgA synthesis [3]. (ii) In addition to the quantitative analysis of the intrathecal immune synthesis, they can also be assessed qualitatively, albeit with greater analytical sensitivity, by a comparison of the patterns of the polyspecific immune reaction in the CSF and serum in isoelectrofocusing (IEF; so called 'olicoglonal IgG bands' or OCB), as correctly pointed out by Sapko et al. [1].This method is currently available exclusively for IgG, although efforts are ongoing to extend it to other immunoglobulin classes. According to a still valid interpretational guideline of the European consensus [4], five patterns of the OCB exist. As a practical tip, IEF can be omitted if $\mathrm{Q}_{\mathrm{IgG}}$ $>\mathrm{Q}_{\mathrm{Alb}}$ or when undoubtful intrathecal synthesis is observed on a Reibergramm. The diagnostic sensitivity of the intrathecal synthesis of IgG in MS is so high that its absence practically excludes (with minor exceptions) a diagnosis of chronic neuroinflammation of the autoimmune type. Its diagnostic specificity, though, is much worse, particularly among differential diagnoses of chronic neuroinflammations.

(iii) Polyspecific immune reaction in the central nervous system is perhaps best reflected (and most commonly misinterpreted) by quantitative analysis of the intrathecal synthesis of the immunoglobulins specific against a vast range of - causatively unrelated - antigens, such as neurotrophic viruses, with antibodies against measles $(\mathrm{M})$, rubella $(\mathrm{R})$, varicella-zoster $(\mathrm{Z})$, and herpes simplex $(\mathrm{H})$ playing the most important diagnostic roles. A simultaneous analysis of CSF-serum specific indices of the antibodies, in the IgG class, against the four viruses is commonly referred to as the 'MRZH Reaction'. This is predictive of autoimmune-type chronic inflammatory disease, even at the first clinical manifestations $[5,6]$.

(iv) A mild, if any, blood-CSF barrier dysfunction is usually observed in MS, with $\mathrm{Q}_{\mathrm{Alb}}$ rarely larger than $25 \times 10^{-3}$. Similarly, mild CSF pleocytosis is frequently observed, but the 
cell count rarely exceeds 30 cells $/ \mathrm{mm}^{3}$, and they are almost exclusively mononuclear. Larger values of one of the two analytes indicate strongly against MS (apart from cases of a comorbidity, of course), hence playing an important differential diagnostic role.

(v) On top of that, in the search for novel candidate biomarkers it is of crucial importance to consider blood-CSF protein diffusion mechanisms, directly related to the issues of protein biophysics discussed above in (i). This is particularly important where a novel candidate CSF biomarker is of mixed blood- and brain-derived origin. Ignoring molecular-sized dependent blood-CSF diffusion, particularly in cases of an even slightly dysfunctional blood-CSF barrier, leads to serious misinterpretation of the source of the candidate biomarker, and consequently its diagnostic validity.

Taken together, we believe that it is extremely important for neurologists, as well as for clinical chemists involved in CSF analysis, to properly understand the role of the CSF in the diagnosis of MS, as well as in a range of other neurological conditions.

The importance of CSF analysis in current neurology is reflected by the diagnostic guidelines published by societies of neurologists, such as that of Germany [7], though unfortunately not (yet) adopted in some countries.

A thorough analysis of routine CSF biomarkers is not only of fundamental help in caring for the patient, but also provides a solid basis for the search for novel candidate biomarkers such as those reviewed by Sapko et al. [1].

\section{References}

1. Sapko K, Jamroz-Wiśniewska A, Marciniec M, et al. Biomarkers in Multiple Sclerosis: a review of diagnostic and prognostic factors. Neurol Neurochir Pol. 2020; 54(3): 252-258, doi: 10.5603/PJNNS. a2020.0037, indexed in Pubmed: 32462652.

2. Reiber H. Flow rate of cerebrospinal fluid (CSF) - A concept common to normal blood-CSF barrier function and to dysfunction in neurological diseases. Journal of the Neurological Sciences. 1994; 122(2): 189-203, doi: 10.1016/0022-510x(94)90298-4.

3. Reiber H, Peter J. Cerebrospinal fluid analysis: disease-related data patterns and evaluation programs. Journal of the Neurological Sciences. 2001; 184(2): 101-122, doi: 10.1016/s0022-510x(00)00501-3.

4. Andersson M, Alvarez-Cermeño J, Bernardi G, et al. Cerebrospinal fluid in the diagnosis of multiple sclerosis: a consensus report. $J$ Neurol Neurosurg Psychiatry. 1994; 57(8): 897-902, doi: 10.1136/ jnnp.57.8.897, indexed in Pubmed: 8057110.

5. Kulakowska A, Mroczko B, Mantur M, et al. Multiplexing analysis of the polyspecific intrathecal immune response in multiple sclerosis. Methods. 2012; 56(4): 528-531. doi: 10.1016/j.ymeth.2012.03.002, indexed in Pubmed: 22445706.

6. Reiber H, Lange P. Quantification of virus-specific antibodies in cerebrospinal fluid and serum: sensitive and specific detection of antibody synthesis in brain. Clin Chem. 1991; 37(7): 1153-1160, indexed in Pubmed: 1855284.

7. Tumani H, Petereit HF, Gerritzen A, et al. S1 guidelines "lumbar puncture and cerebrospinal fluid analysis" (abridged and translated version). Neurological Research and Practice. 2020; 2(1), doi: 10.1186/ s42466-020-0051-z. 